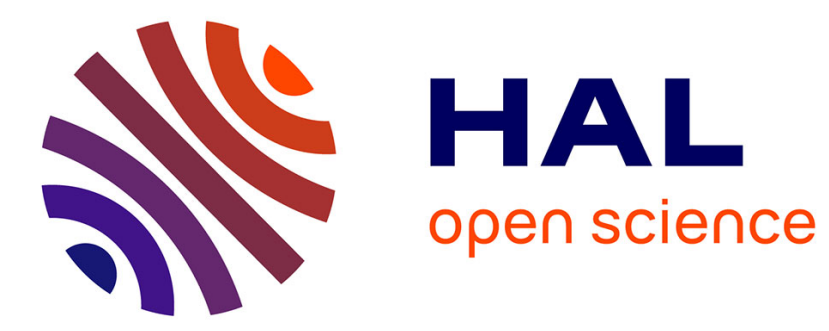

\title{
Le défi internet: l'industrie du disque américaine à l'heure du numérique
}

Claude Chastagner

\section{To cite this version:}

Claude Chastagner. Le défi internet: l'industrie du disque américaine à l'heure du numérique. Revue Française d'Etudes Américaines, 2005, 104 (juin), pp.63-73. halshs-00176144

\section{HAL Id: halshs-00176144 \\ https://shs.hal.science/halshs-00176144}

Submitted on 2 Oct 2007

HAL is a multi-disciplinary open access archive for the deposit and dissemination of scientific research documents, whether they are published or not. The documents may come from teaching and research institutions in France or abroad, or from public or private research centers.
L'archive ouverte pluridisciplinaire HAL, est destinée au dépôt et à la diffusion de documents scientifiques de niveau recherche, publiés ou non, émanant des établissements d'enseignement et de recherche français ou étrangers, des laboratoires publics ou privés. 


\section{Le défi Internet : \\ L'industrie du disque américaine à l'heure du changement}

\section{Abstract :}

The American record industry claims that Internet is threatening its very existence, particularly because of illegal downloading. This article explores to what extent this claim is true, what other elements challenge the hegemony of the major record companies, and focuses on the new business models made necessary by the development of Internet.

Mots clés:

Internet, piratage, téléchargement.

II y a eu au cours de l'année 2003 plus de 15 milliards de fichiers musicaux (simples chansons ou albums entiers) échangés de manière illégale à travers Internet . On sait déjà que ce nombre sera en très forte augmentation dans les années à venir. Et pourtant, les ventes de CD, en baisse depuis ces cinq dernières années, ont augmenté de 10,2\% aux États-Unis au cours des six premiers mois de 2004 (289 millions contre 267 millions pour la même période en 2003). Les concerts, quant à eux, représentent une source toujours croissante de profits. ${ }^{1}$ Ces simples chiffres donnent une idée de la complexité de la situation et incitent à relativiser les propos alarmistes des majors de l'industrie du disque, Universal, Sony/BMG (Bertelsmann Music Group, Warner et EMI (Electrical \& Musical Industries). ${ }^{2}$

Nous nous proposons, dans cet article, d'examiner les changements qu'Internet en train d'imposer au marché du disque américain. Depuis plusieurs années, les médias se sont fait l'écho des réactions hostiles des milieux du disque face au piratage individuel. Mais la copie illégale n'est qu'un aspect du problème. Internet modifie également les principes de distribution et commercialisation de la musique. Après un bref rappel historique des rapports conflictuels que l'industrie du disque a eu avec les nouvelles technologies, nous montrerons ce que la situation actuelle a d'inédit et en quoi elle amène une modification du comportement des différents acteurs. Dans un deuxième temps, nous tenterons d'analyser les autres facteurs qui peuvent expliquer la mutation que connaît le marché du disque.

Dès les années 1920, l'apparition des radios commerciales provoqua une chute des ventes de disques, déclenchant ainsi les premières tentatives de l'industrie de protéger son marché face à l'arrivée d'une nouvelle technologie. Les artistes furent contraints de signer des contrats leur interdisant de travailler pour la radio, mais seule l'amélioration de la qualité sonore des disques permit d'enrayer de façon durable la chute des ventes. Le même scénario se répéta en 1933 avec l'avènement 
des radios à modulation de fréquence. L'industrie fit cette fois-ci pression sur la Federal Communications Commission pour tenter de limiter la propagation de ce nouveau type de radios. Ruiné par les procès intentés par la RCA (Radio Corporation of America), Edwin Armstrong, inventeur de la Frequency Modulation (FM), se suicida.

En 1964, c'est la commercialisation de la cassette audio qui inquiéta fortement les majors. Avec un coût deux fois moindre que celui d'un disque, la cassette offrait pour la première fois à tout auditeur la possibilité de copier de la musique. Lorsqu'à la fin des années 1970 les ventes de disques chutèrent de nouveau, les compagnies lancèrent une campagne contre la copie privée. Ils exigèrent une taxe sur la vente des cassettes vierges pour compenser le manque à gagner des ventes de disques. Le lancement du Walkman par Sony ne fit qu'aggraver la situation. C'est l'arrivée du Compact Disc qui relança provisoirement les ventes. Séduits par la qualité sonore de ce nouveau support, de nombreux amateurs de musique décidèrent en effet de remplacer leur collection de disques traditionnels (en vinyle) par des CDs. En 1987, un nouvelle menace se profila avec la Digital Audio Tape (DAT) qui permet une duplication numérique à l'infini, sans aucune perte de qualité. Le débat juridique qui accompagna la sortie de la DAT empêcha sa diffusion dans le grand public. En 1992, I'Audio Home Recording Act instaura la taxe sur les cassettes vierges que l'industrie réclamait depuis les années soixante-dix. La même année eut lieu la première action en justice intentée contre le réseau numérique Compuserve qui permettait à ses utilisateurs de télécharger des fichiers musicaux sans qu'il soit nécessaire d'obtenir le consentement des propriétaires des droits ou de leur payer une redevance. Depuis lors, avec la démocratisation d'Internet et les progrès technologiques permettant la transmission de plus en plus rapide et fiable de fichiers, le phénomène de la copie illégale par Internet ne cesse de s'amplifier et suscite des réactions le plus souvent agressives de la part de l'industrie du disque.

\section{Internet, une nouvelle donne}

Il est tentant de ne voir dans l'affrontement actuel qu'une répétition des précédents. Néanmoins, pour la première fois, tout auditeur a la possibilité de dupliquer une chanson à l'infini, de la diffuser à l'échelle mondiale et d'accéder à une audiothèque riche de millions de morceaux. En cela, l'industrie du disque est confrontée à une situation inédite, qui remet fondamentalement en cause le modèle économique sur lequel elle est fondée. De plus, toutes les tentatives de l'industrie pour contrer cette forme de piratage se sont soldées par des échec retentissants sur le plan des relations publiques, échecs amplifiés par la prodigieuse réactivité d'Internet quant à la diffusion de l'information. 
La Recording Industry Association of America (RIAA), le puissant syndicat de l'industrie du disque américaine, définit quatre formes de piratage:

- les reproductions illégales (pirate recordings) à l'échelle industrielle, où seul le son est dupliqué;

- les contrefaçons (counterfeits), là encore à l'échelle industrielle, où le produit complet (CD et pochette) est contrefait;

- les enregistrements pirates (bootlegs) de concerts ou d'émissions radiophoniques à l'échelle individuelle ou industrielle;

- le piratage Internet (online piracy) à l'échelle individuelle, même si les fichiers ainsi échangés peuvent s'inclure dans un trafic de grande envergure.

Même si, de son propre aveu, le piratage individuel n'est qu'une des causes avancées par la RIAA pour les quatre milliards de dollars de perte dues au piratage, c'est sur lui que portent les efforts de répression les plus médiatisés.

L'utilisation d'Internet pour l'acquisition illégale de musique est en constante modification. Alors que les premiers échanges de fichiers se faisaient par l'intermédiaire de serveurs, la transmission se fait aujourd'hui directement d'utilisateur à utilisateur, sur le principe du P2P (peer to peer). Ce mode d'échange décentralisé, n'offrant plus de responsable unique (l'opérateur du serveur), est plus difficile à contrer. En 1999, le premier logiciel P2P, le célèbre Napster, était encore basé sur un serveur central qui contenait les listes des fichiers mis à disposition par ses utilisateurs, et non les fichiers eux-mêmes. Par l'intermédiaire de la RIAA, les majors intentèrent une série de procès au propriétaire et fondateur de Napster qui entraînèrent la fermeture du site en 2001. Napster connaîtra une deuxième vie, légale celle-ci, suite à l'accord signé avec les majors le 5 juin 2001 par lequel il devient une filiale de MusicNet, un service d'abonnement musical en ligne créé par une entreprise de Seatlle, RealNetworks, en accord avec les compagnies de disques AOL Time Warner, BMG et EMI. Napster finit par déposer son bilan le 3 juin 2002. Les autres systèmes, non centralisés, qui ont depuis remplacé Napster (Kazaa, Limewire, Edonkey) et sur lesquels se fait aujourd'hui l'essentiel des échanges illégaux, s'avèrent beaucoup plus difficiles à neutraliser. Les tentatives de la RIAA pour les contrer ne manquent pourtant pas.

En octobre 2001, la RIAA attaque en justice Kazaa, Morpheus et Grokster, au niveau fédéral, mais le juge Stephen Wilson, la déboute au titre que ces sociétés n'enfreignent pas les lois du copyright. Depuis, la société qui diffuse Kazaa a déplacé son siège social à Vanuatu, paradis fiscal du Pacifique. La RIAA s'est également attaquée aux utilisateurs individuels de ces logiciels. Plusieurs milliers d'entre eux (6191 exactement de septembre 2003 à novembre 2004) ont été assignés en justice. Les bénéfices de ces procédures restent incertains. Si les risques encourus sont désormais plus tangibles, ces actions ont surtout contribué à 
l'érosion de l'image des multinationales du disque. Une autre stratégie a été de décourager les utilisateurs en diffusant des fichiers "leurres" sur les réseaux P2P. Les déconvenues répétées des internautes étaient censées les inciter à se tourner vers des formes légales d'acquisition de musique, de l'achat traditionnel en magasin au téléchargement sur des sites gérés par les compagnies de disques ou leurs filiales. Quelle a été l'efficacité de ces actions? D'après une étude publiée en 2004, I'utilisation des réseaux P2P est en constante augmentation: "we challenge the stated P2P reports [that traffic is dropping] and their conclusions, emphasizing the fact that measurements of P2P traffic are problematic." (Karagiannis) Ils soulignent dans leur conclusion que les actions de la RIAA n'ont pas eu d'impact significatif sur les pratiques des "pirates" (augmentation de cinq millions du nombre des utilisateurs de P2P en 2004, pour un total estimé à 23 millions en juin 2004 (NYT, Op-Ed).

En parallèle, la RIAA s'est attaquée au problème en amont, en tentant de protéger les CDs contre la lecture (et donc la copie potentielle) sur ordinateur. Malheureusement pour la RIAA, outre les questions légales liées à la restriction des possibilités de lecture des disques achetés dans le commerce en toute légalité, les méthodes mises en oeuvre se sont le plus souvent avérées facilement contournables (par exemple avec une simple trace de feutre sur la bordure du CD prétendument protégé). Là encore, l'image publique de la RIAA en a pâti. Au niveau législatif enfin, la RIAA a cherché à faire passer une loi l'autorisant à fouiller le contenu d'un ordinateur personnel sans autorisation préalable et sans que son propriétaire en soit prévenu, remettant en cause la protection de la vie privée. Cette loi aurait constitué un complément à l'USA Act (la loi anti-terroriste votée après le 11 septembre), mais la démarche n'a finalement pas abouti. Cette initiative n'a pas non plus manqué d'être très critiquée par la communauté Internet.

De leur côté, la réaction des artistes a été partagée. Certains, comme Metallica et $\mathrm{Dr}$ Dre, ont très tôt pris position contre la copie illicite, à la grande déception de leur fans. La RIAA a obtenu le soutien d'artistes réputés pour une vaste campagne publicitaire en faveur de l'acquisition de musique par des moyens légaux dont le message indiquait: "I support the RIAA and its actions against Music Archive Sites on the Internet because copyright is my lifeline, without it recording artists would drown. Don't trash us by pirating sound recordings on the Net. Get real. Get legit". De telles recommandations de la part d'artistes richissimes, tels que Paul McCartney ou Mick Jagger, n'ont eu qu'une portée limitée.

D'autres en revanche, tels que Robbie Williams ou Prince soutiennent le développement d'Internet comme moyen de circulation de la musique. Pour eux, les véritables pirates sont les majors, et leurs victimes sont aussi bien les artistes que les consommateurs. C'est le sens de l'intervention de Courtney Love, célèbre artiste de rock et de cinéma, le 16 mai 2000, lors de la Digital Hollywood Online 
Entertainment Conference à New York: "It's piracy when those guys that run those companies make side deals with the cartel lawyers and label heads so that they can be 'the labels' friend', and not the artists'. Piracy is the act of stealing an artist's work without any intention of paying for it" affirme-t-elle, avant d'ajouter que c'est exactement ce que font les compagnies de disques (NYT, "Is it theft").

D'autres vont plus loin encore et utilisent la duplication à partir d'Internet comme outil promotionnel. Ainsi Wilco, un groupe de Chicago, a diffusé son album Yankee Hotel Foxtrot en ligne gratuitement suite à la rupture de leur contrat par leur maison de disque en 2001. Lorsque l'album a été commercialisé quelque temps après, il s'est classé dans les listes de ventes à une bien meilleure position que les précédents, preuve que le téléchargement gratuit ne nuit pas forcément aux ventes. On peut avancer comme raison que le goût pour la possession d'un objet physique, le disque, n'a pas complètement disparu. Le leader du groupe, Jeff Tweedy, raconte également qu'après avoir obtenu l'album gratuitement, beaucoup de fans ont cherché à rémunérer directement le groupe, acte symbolique qui prouve a contrario de l'attitude des majors, que ne pas traiter les utilisateurs en voleurs potentiels permet de se créer une image très favorable vis à vis du public.

\section{Les facteurs de mutation du marché de la musique.}

Le piratage, privé ou industriel, n'est pas le seul responsable des bouleversements que connaît l'industrie du disque, même si Internet reste la cause centrale. Pour commencer, le principal acteur du marché, c'est-à-dire le consommateur, change de comportement. La musique ne représente plus le principal lien social entre les jeunes comme elle l'était dans les années 1960 et 1970. Elle n'est également plus la principale source de distraction comme elle l'était dans les années quatre-vingt. Le DVD, les jeux vidéos et les nouveaux moyens de communication (Internet, téléphone portable) se sont taillés une part importante dans le budget et le temps libre des adolescents. Ainsi, il est intéressant de noter que la vente des DVDs est en augmentation constante (101\% de plus en 2004, passant de 5,6 à 11,2 millions d'unités en un an, pour une augmentation de $54 \%$ en valeur).

Les majors se sont également forgé une image publique qui contribue à dévaloriser les "produits" qu'elles vendent. Leur forte concentration (4 majors seulement se partagent $90 \%$ des ventes) a éliminé le rapport de fidélité que le consommateur pouvait avoir avec elles via des labels indépendants respectés (Blue Note, Stax, Atlantic, Motown, Charisma, Island, Virgin à ses débuts...).

Par ailleurs, les majors sont toutes cotées en bourse, ce qui leur impose une pratique commerciale allant vers une rentabilité à brève échéance, au détriment d'investissements à plus long terme dans la carrière d'un artiste. Richie Sambora, musicien réputé, décrit ainsi la situation: "Before, they would let an artist find himself 
and go through a period of making their mistakes and learning from their mistakes. You don't have a margin for that at this point". (Frontline). De même, pour limiter les risques pris à l'investissement, les artistes ressemblant à ceux au succès déjà établi sont privilégiés. Les points de ventes eux-mêmes imposent des contraintes au marché. Les gros magasins comme Best Buys, Wal-Mart ou Target représentent $50 \%$ des ventes réalisées par les majors, mais ces ventes ne constituent qu'entre 1 et $3 \%$ du chiffre d'affaire de ces "Big Box Retailers". Ils n'offrent qu'un espace de rayonnage limité, ce qui oblige les majors à ne leur fournir que des disques récents dont les ventes sont assurées, alors que c'est avec les anciens titres déjà amortis que les compagnies ont leur marge la plus élevée. ${ }^{3}$ L'hégémonie des "Big Box Retailers" a entraîné la fermeture de grandes chaînes de magasins de disques comme Tower Records, garants d'une certaine diversité musicale.

Cette diversité a été aussi entamée par le processus de restructuration du paysage radiophonique américain. Les ventes d'un artiste sont directement liées à son exposition au public. Tant qu'il existait aux États-Unis des milliers de stations de radio locales, la liberté de programmation dont chacune disposait assurait là encore une grande diversité de l'offre musicale. La loi de 1996 a supprimé la limite de 40 stations qu'un seul propriétaire peut posséder à l'échelle du pays. Aujourd'hui, Clear Channel, Cox et Infinity possèdent à eux trois l'essentiel des stations de radio des États Unis et leurs imposent une "play list" limitée parfois à 14 chansons différentes par semaine. Même si les avis sont partagés, il est permis de penser que cela conduit à un appauvrissement du choix musical offert par les majors. Cela accentue leur perte de respectabilité, déculpabilisant ainsi l'utilisateur de P2P qui n'a pas la sensation de voler une multinationale dont il désapprouve a priori l'existence, voire même de faire "oeuvre de justice", tant il est couramment admis que les maisons de disques exploitent leurs artistes (voir Chastagner, Rebels). D'après les calculs effectués par Steve Albini, célèbre producteur de rock indépendant, même un groupe ayant vendu 250000 albums se retrouverait à devoir $\$ 14000$ à sa maison de disque pour rembourser les frais de studio, de promotion et de tournée, tandis que la maison de disque aurait encaissé 710000 dollars, et l'industrie du spectacle dans son ensemble plus de 3 millions de dollars.

Enfin, la copie numérique est en elle-même un marché très lucratif : les ventes de graveurs de CD, et surtout de lecteurs MP3, explosent. La possibilité de télécharger librement de la musique est clairement utilisée comme argument publicitaire par les fournisseurs d'accès Internet, ce qui contribue encore à décriminaliser le téléchargement. Le Nouvel Observateur révèle que "selon une étude du Royal Institute of Technology suédois, le téléchargement occupe $40 \%$ du temps de connexion des internautes dans le monde et constitue bien la première motivation d'abonnement à l'Internet rapide". 
Internet représente donc un changement fondamental de modèle commercial par rapport aux majors. II offre d'abord au consommateur une souplesse de service accrue : celui-ci n'est plus contraint d'acheter un album entier lorsqu'il ne souhaite avoir que quelques morceaux. De plus, la disponibilité est quasi immédiate (quelques minutes pour une chanson à travers une connexion haut débit). Plutôt que d'acheter légalement un album complet dont il sait que les autres chansons constituent souvent du "remplissage", le consommateur préfère donc ne télécharger que les chansons qui l'intéressent. Enfin l'offre extrêmement diversifiée d'Internet (800 millions de fichiers musicaux disponibles en juin 2004) permet aux utilisateurs de P2P de retrouver facilement les œuvres des artistes qu'ils aiment, indépendamment de leur niveau de vente, mais aussi de partager les disques qu'ils apprécient, leur permettant ainsi de constituer des liens avec d'autres amateurs de musique ayant les mêmes goûts. Internet constitue donc un vecteur culturel unique.

\section{Quel avenir se profile pour le marché du disque américain ?}

Les premiers sites Internet offrant un accès légal à de la musique en ligne n'ont pas rencontré un franc succès : Pressplay, MusicMatch et Rhapsody, basées sur des formules d'abonnement, ne totalisent à eux trois que 50000 abonnés. L'évolution la plus remarquable est certainement iTunes, site de vente de musique en ligne créé par Apple en avril 2003, qui fonctionne en parallèle avec le iPod, lecteur MP3 le plus vendu actuellement, également fabriqué par Apple et sorti début 2002 (1,5 millions d'unités vendues dès la première année, malgré un prix de vente autour de \$300). Actuellement, $70 \%$ des téléchargements légaux sont effectués sur iTunes, avec un pic de 1,3 million clients en avril 2004. Depuis, le nombre de clients a légèrement diminué (1 million par mois), ce qui semble correspondre à la fin de la période d'offres promotionnelles. Plus de 70 millions de morceaux ont été vendus sur iTunes depuis sa création, à comparer néanmoins avec les 12 milliards de morceaux vendus par an sur CD dans le circuit conventionnel. Une chanson sur iTunes coûte 99 cents, et un album $\$ 10$. Les fichiers ainsi téléchargés ne sont en théorie copiables que suivant certaines restrictions relativement peu contraignantes.

Pour expliquer le succès de son produit par opposition à ses premiers concurrents, Steve Jobs, PDG d'Apple, insiste sur le désir qu'a le consommateur de "posséder" sa musique, plutôt que d'en être seulement le dépositaire temporaire. En cela, il va à l'encontre du modèle identifié par Jeremy Rifkin dans The Age of Access, d'une société de consommation de plus en plus axée sur un système d'abonnement. Notons au passage que si le succès du téléchargement confirme l'indifférence croissante de l'auditeur envers un support matériel, il n'en reste pas moins que la moitié des achats sur iTunes concerne des albums dans leur intégralité et que l'on observe un déplacement de l'intérêt du consommateur du disque vers le support (en 
l'occurrence l'iPod). D'autres sites suivant le même modèle qu'iTunes sont apparus depuis, tels que MSN Music (qui appartient à Microsoft), Virgin Digital ou Music Match, mais ils souffrent de ne pas offrir un support séduisant tel que l'Ipod d'Apple. La chaîne de supermarchés Wal-Mart a également créé son propre site de téléchargement.

A mi-chemin entre ces sites de téléchargement légaux et le P2P pirate apparaît un nouveau développement : des réseaux $\mathrm{P} 2 \mathrm{P}$ sur lesquels ne se trouvent que des morceaux autorisés. Plusieurs accords ont récemment été signés entre les majors et des sociétés gérant des réseaux P2P (Machboxx, Wippit). C'est avec Peerlmpact que les accords sont les plus avancés, puisque Universal, Warner et Sony BMG ont déjà signé, et qu'EMI est en attente. Le service devrait ouvrir début 2005, chaque chanson coûtera 99 cents et sera protégée contre la duplication, protection sans doute illusoire comme le montrent les expériences précédentes. Quelle différence alors avec iTunes ? Greg Kerber, PDG de Peerlmpact, pense que les utilisateurs seront attirés par le concept de communauté, en l'occurrence une communauté de fans (ou plus prosaïquement de consommateurs d'un même produit) : "We have seen that when successful companies like ebay build large communities, and people share in that, there is power in that for the consumer" (CNet News).

Autre initiative des majors, le lancement de nouveaux groupes uniquement à travers Internet, comme c'est le cas avec "The Shazam" par Universal. L'investissement nécessaire étant moins important que dans le cas d'un lancement classique et les risques d'invendus étant nuls, les majors voient là un moyen plus profitable et moins risqué de lancer de nouveaux groupes. L'Electronic Frontier Foundation a de son côté publié un livre blanc, "Let The Music Play", qui propose le principe d'un paiement volontaire mensuel de $\$ 5$ qui assurerait aux internautes la possibilité de télécharger autant de fichiers musicaux qu'ils le souhaitent sans restriction et sans risque de poursuites judiciaires. Les sommes ainsi collectées (des calculs préliminaires prévoient 3 milliard de dollars par an) seraient réparties par l'intermédiaire d'un organisme central entre les différents artistes en fonction de leur popularité. Ce fonctionnement est à rapprocher du système actuel de gestion des droits d'auteurs par des compagnies comme ASCAP (American Society of Composers, Authors, and Publishers), BMI (Broadcast Music Inc.)) ou SESAC (Society of European Stage Authors and Composers), équivalents de notre SACEM (Société des Auteurs, Compositeurs et Editeurs de Musique).

Il semble donc désormais établi que le téléchargement, légal ou non, ne s'arrêtera pas et qu'Internet va continuer de jouer un rôle croissant dans la consommation de musique des Américains. Les majors semblent donc contraintes de s'y adapter pour 
9

ne pas disparaître au profit de ceux qui auront su créer un modèle économique exploitable sur ce nouveau média.

Claude Chastagner, Université Paul Valéry, Montpellier III

Guillaume Laurent, Ingénieur informaticien, Sophia Antipolis 


\section{Notes}

1. The Economist indique que d'après son manager, une des plus grandes stars de la chanson américaine aurait gagné l'année dernière 9 millions de dollars grâce aux ventes de ses CDs, 3 millions grâce aux produits dérivés, 15 millions par les films et 15 millions rien que par ses concerts. (The Economist).

2. The Economist révèle qu'une étude interne réalisée pour le compte d'une major admet que $75 \%$ de la baisse des ventes de CD aux États-Unis n'a rien à voir avec le piratage sur Internet. (The Economist).

3. Or, le fait de ne rechercher que des succès à court terme plutôt que de construire un fonds de catalogue ne peut que fragiliser à moyen terme la situation des majors. 


\section{Bibliographie}

Chastagner, Claude. "Rebels on the Net", in Cercles, <www.cercles.com>, Université de Rouen, $n^{\circ}$ 3, mars 2001

CNet News. http://news.com.com/2102-1026_3-5465845.html?tag=st.util.print

Economist, The. http://www.economist.com/displaystory.cfm?story_id=3329169

Frontline. http://frontline.com. Transcript de l'émission diffusée sur PBS le 27 mai 2004.

Kariagiannis, Thomas, et al. "Is P2P dying or just hiding". in IPTPS 03, 2004.

New York Times, The. "Is It Theft, or is it Freedom? 7 Views of the Web's Impact on Culture Clashes", 7/9/2000, C42.

---.25 juin 2004, Op. Ed. page

Nouvel

Observateur, Le.

http://archives.nouvelobs.com/recherche/article.cfm?id=12141

Rifkin, Jeremy. The Age of Access. The New Culture of Hypercapitalism where All of Life is a Paid-for Experience. New York : Putnam, 2000. 